\title{
Cost Effective Synthesis of Bulk Thermoelectric Higher Manganese Silicide for Waste Heat Recov- ery and Environmental Protection
}

\author{
Xinghua Shi, Zahra Zamanipour, Arash Mehdizadeh Dehkordi, Kenneth F. Ede, Jerzy S. Krasinski, Daryoosh \\ Vashaee* $^{*}$ \\ Helmerich Advanced Technology Research Center, Oklahoma State University, Tulsa, OK 74106, USA \\ "daryoosh.vashaee@okstate.edu
}

\begin{abstract}
Higher manganese silicide (HMS) is a useful thermoelectric material for waste heat recovery in medium to high temperature range. It is made from two of the most abundant materials on earth. Moreover, it is non-toxic and environmentally responsible. A low-cost, scalable, and quick method of synthesizing bulk thermoelectric higher manganese silicide is proposed for its industrial manufacturing. Heat treatment alloying of higher manganese silicide powder is proposed for producing its large quantity in a cost effective way. The process involves heat treatment of the elemental powders at $1050{ }^{\circ} \mathrm{C}$ for 1 hour. Thermoelectric properties of the resultant samples were studied and compared with the samples made by mechanical alloying. In comparison, both methods result in similar trends in thermoelectric properties however with significant cost reduction and ease of fabrication for the proposed method.
\end{abstract}

Keywords- thermoelectric, higher manganese silicide, synthesis, heat treatment

\section{INTRODUCTION}

Due to recent global environmental issues, increases in fuel prices, and exhaustion of fossil fuels the demand for new sources of energy has rapidly increased. Thus, the development of clean, recycled, environmentally friendly and lowcost energy sources is an important and challenging issue. Thermoelectric (TE) generators can convert waste heat energy to electricity. With about $60 \%$ of the world's useful energy wasted as heat, they offer useful means to recycle large amounts of energy.

For green applications the most well-known thermoelectric materials based on groups IV and V chalcogenides, have several drawbacks such as cost and toxicity. In contrast, Manganese and Silicon are non-toxic and abundant elements in the Earth's crust. Higher Manganese Silicide (HMS) is a good candidate to make low-cost thermoelectric materials with lowenvironmental issues [1] for medium to high temperature (400 ${ }^{\circ} \mathrm{C}-700{ }^{\circ} \mathrm{C}$ ) applications. The development of high performance thermoelectric generators for this range of temperature is highly desired [2]. In addition, HMS offers a low vapor pressure, strong hardness and high resistance to oxidation with atmospheric oxygen [3-4]. One of the most immediate large scale applications of HMS is waste heat recovery in automobiles. In most automobiles, more than 70 percent of the fuel's energy is wasted as heat. HMS thermoelectric generators can be used in automobiles to recover the waste heat from the engine and increase the fuel efficiency. Thus, it is a prospec-

This report is partially based upon work supported by Air Force Office of Scientific Research (AFOSR) High Temperature Materials program under grant no. FA9550-10-1-0010 and the National Science Foundation (NSF) under grant no. 0933763. tive means of using the waste heat discharged from energy systems such as automobiles, combined cycles and waste material incineration systems [5].

Higher manganese silicides a $\mathrm{MnSi}_{\mathrm{x}}$ with $\mathrm{x}$ in the range from 1.73 to 1.75 have been studied as one of the promising p-type thermoelectric materials. There are several reports concerning different tetragonal phases that include $\mathrm{Mn}_{11} \mathrm{Si}_{19}$, $\mathrm{Mn}_{15} \mathrm{Si}_{26}, \mathrm{Mn}_{27} \mathrm{Si}_{47}$ and $\mathrm{Mn}_{4} \mathrm{Si}_{7}$ [6-7]. HMS has almost similar thermoelectric properties and they all make Chimney-ladder structures with an energy gap of approximately $0.7 \mathrm{eV}$.

The preparation of HMS alloys for thermoelectric applications is quite challenging. There are different methods to produce HMS alloys. One such method is synthesizing HMS alloy through vacuum melting of the elemental materials [8]. A disadvantage is the compositional contamination due to the complexity of the phase formations of $\mathrm{Mn}-\mathrm{Si}$ at high temperature. In addition, the high vapor pressure of manganese at elevated temperatures makes the controlled composition of $\mathrm{Mn}$ and Si difficult in this process. High energy mechanical alloying is an alternate method of producing HMS thermoelectric materials. Mechanical alloying can minimize compositional heterogeneity and produce powder for making bulk materials. However, mechanical alloying requires a relatively long milling time which consumes significant electrical energy [9].

Alternatively, we have introduced a new process with fewer challenges to synthesize higher manganese silicide in this paper. Heat treatment of the mixed powder was used to produce large amounts of powder in higher manganese silicide phases. This offers a relatively easy, low-cost, efficient and rapid method to produce higher manganese silicide compared with other existing methods. Optimizing the temperature and time for heat treatment and making comparison of this process with mechanical alloying methods are the concerned subjects of this report.

\section{METHODOLOGY}

Silicon (100 mesh, 99.9\%, Alfa Aesar) and manganese (325 mesh, 99.99\%, Alfa Aesar) were weighed in an argon-filled glove box for composition of $\mathrm{MnSi}_{\mathrm{x}}$ with $\mathrm{x}=1.75$. The mixed powder was loaded under an argon atmosphere in tungsten carbide bowls with a ball to powder weight ratio (BPR) of 5 . The bowl was hermetically sealed and the powder was milled in a planetary ball mill (Fritsch, P6) at $650 \mathrm{rpm}$ for 159 hours for complete alloying. A second mixed part of higher manganese silicide powder was milled for three hours and subsequently heat treated at $1050{ }^{\circ} \mathrm{C}$ in a muffled furnace under 
argon atmosphere for 1 hour. Alloying process was investigated by XRD diffraction after heat treatment. The prepared powder was weighed and loaded into the graphite die with an inner diameter of $12.7 \mathrm{~mm}$. The samples were sintered under $108 \mathrm{MPa}$ pressure at $950{ }^{\circ} \mathrm{C}$ for $4-5$ minutes. The sintered samples were cut into disks and rods for characterizations. The thermal conductivity was measured by a laser flash apparatus (Netzsch LFA 457 Micro Flash). The Seebeck coefficient and electrical conductivity were measured simultaneously using a commercial apparatus (Ulvac-Riko's ZEM-3).

\section{EXPERIMENTAL DATA}

X-ray diffraction patterns of HMS alloys synthesised from both methods are shown in Fig. 1. The bottom (black) curve shows the XRD pattern of higher manganese silicide powder using mechanical alloying. This powder was produced by high energy ball milling, with BPR of 5 at 650 RPM for 159 hours. The top (red) curve shows higher manganese silicide powder using heat treatment alloying. As previously addressed, the elemental powders were milled in order to mix them completely and heated at $1050{ }^{\circ} \mathrm{C}$ for 1 hour. The HMS peaks are formed in both spectrums and there is no elemental $\mathrm{Mn}$ or $\mathrm{Si}$ left in the powder. This indicates that the alloying is complete within the accuracy of the XRD analysis. The difference of the grain size between these two spectrums is obvious. It means that by annealing the mixed powder in one hour, HMS can be synthesized in a much shorter time than mechanical alloying.

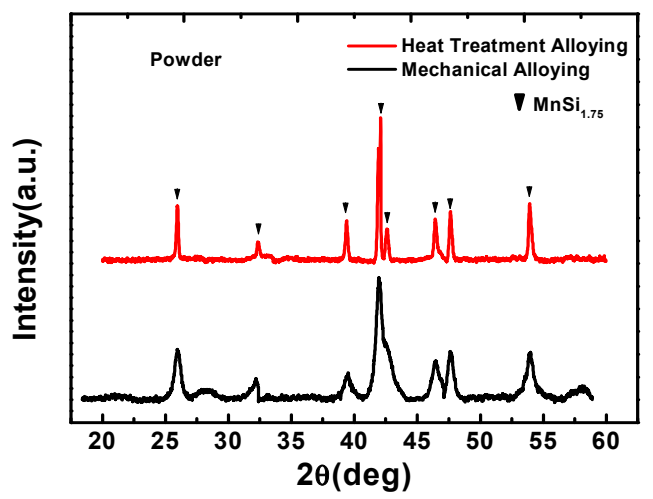

Figure 1: XRD patterns of HMS powder from mechanical alloying (bottomblack) and heat treatment alloying (top-red).

Both powders were sintered by a hot pressing method under the same conditions. The heat was produced by Joule heating the samples by running DC current through the samples. The temperature dependence of transport properties for both samples were measured and compared with each other. The squared curves describe the results for samples made of the mechanical alloyed powder and the circled curves describe the results for samples made of the heat treated alloyed powder.

\section{RESULTS AND DISCUSSION}

In subsequent plots, the squared data points depict the results for samples made of the mechanically alloyed powder.
The circled data points show the results for samples made from the heat treated alloyed powder.

Fig. 2(a) shows the electrical conductivity of the bulk higher manganese silicide samples. The temperature dependence of electrical conductivities demonstrates a metallic or degenerate electrical conduction.
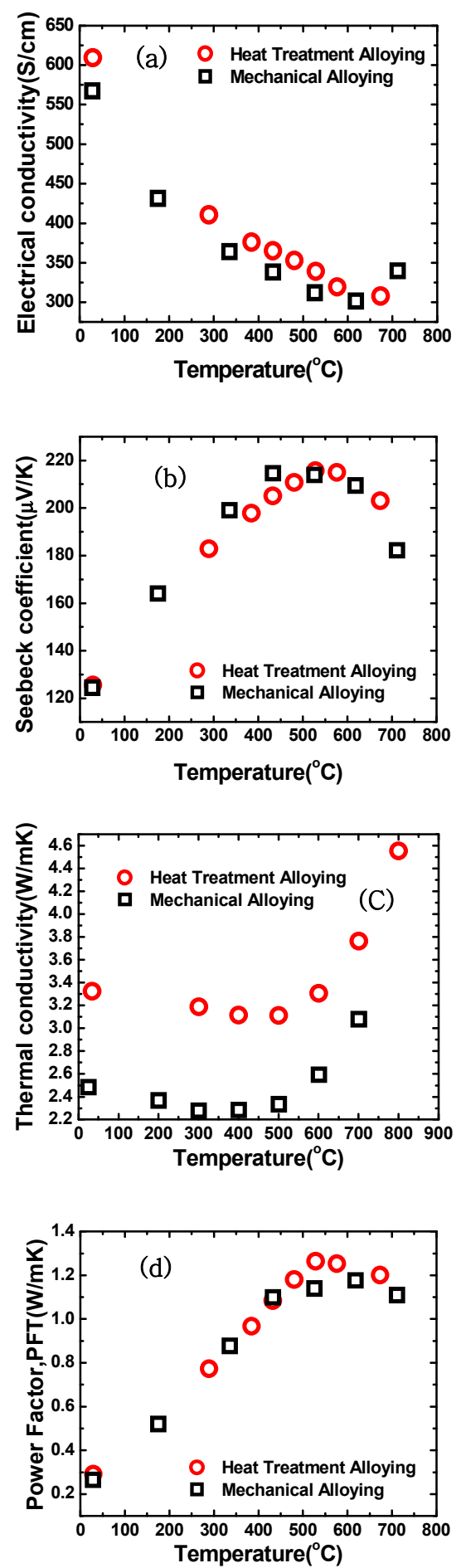


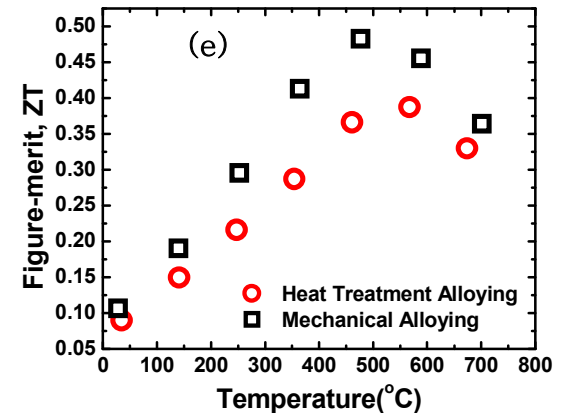

Figure 2: Temperature dependence of (a) electrical conductivity, (b) Seebeck coefficient, (c) power factor, (d) thermal conductivity, and (e) figure-of-merit ZT for mechanically alloyed (squared) and heat treated alloyed (circled) bulk higher manganese silicide samples.

The electrical conductivities of these two samples have only a small difference. The sample made of heat treated alloyed powder has higher electrical conductivity which is due to the larger grain size of this powder. In this sample, electron mean free path is higher due to less grain boundary scattering, hence, increasing the mobility of charge carriers. Therefore electrical conductivity will be enhanced.

Fig. 2(b) shows temperature dependence of Seebeck coefficient for both samples. The Seebeck coefficient versus temperature shows nearly the same values. The Seebeck coefficient increases with temperature up to $550^{\circ} \mathrm{C}$, and decreases at $\mathrm{T}>550^{\circ} \mathrm{C}$ due to the excitation of intrinsic carriers.

According to Fig. 2(c), the temperature dependences of power factor are also similar as expected because the electrical conductivity and Seebeck coefficient of the two alloying methods are similar. The maximum power factor multiply temperature $\left(\mathrm{S}^{2} \sigma \mathrm{T}\right)$ is $1.2 \mathrm{~W} / \mathrm{mK}$ at $550{ }^{\circ} \mathrm{C}$ which is similar to typical HMS thermoelectric samples.

Temperature dependence of thermal conductivity for both samples is shown in Fig. 2(d). Thermal conductivities for the two samples are slightly different. The decrease in thermal conductivity with temperature at $\mathrm{T}<500{ }^{\circ} \mathrm{C}$ is due to reduction in lattice thermal conductivity with temperature.

The temperature dependence of thermal conductivity displays an intrinsic behaviour at $\mathrm{T}>500{ }^{\circ} \mathrm{C}$ due to ambipolar thermal diffusion, which increases the thermal conductivity at high temperatures. The thermal conductivity values for samples made of mechanically alloyed powder is somewhat smaller than samples made using heat treated alloyed powder. This is mainly due to the nanostructure configuration of the mechanically alloyed sample. Due to the significantly increased interfaces of the grain boundaries in this sample, phonons are significantly scattered.

Fig. 2(e) shows the dimensionless figure-of-merit ZT versus temperature for both samples. It is noted that utilizing a heat treated alloying method results in only slightly smaller ZT values compared to a mechanically alloyed method. The peak $\mathrm{ZT}$ in the heat treated alloyed sample is $\mathrm{ZT}=0.4$, and that of the mechanically alloyed sample is $\mathrm{ZT}=0.47$. The somewhat larger electrical conductivity of the heat treated sample (Fig. 2-a) has compensated its larger thermal conductivity (Fig. 2-d) to some extent resulting in close ZT as that of the mechanically alloyed sample. However, the heat treated alloying method has significant advantage in both production cost and time. We used pure HMS without doping for the proof of concept demonstration. The ZT can be enhanced by introduction of dopants to the initial powder and following similar heat treatment process. This would make the proposed technique better suited for large scale production of high quality higher manganese silicide alloy.

\section{CONCLUSION}

In summary, heat treatment alloying of HMS powder was proposed and demonstrated as cost effective means for large scale production of high purity alloyed powder. Although mechanical alloying can also produce pure higher manganese silicide compared to other methods in terms of phase purity, the conventional high energy ball milling process can produce relatively small amount of powder and consumes large electrical energy. We have studied heat treatment of powders to produce pure HMS alloys and made comparisons between these two methods. The heat treatment is carried out at $1050{ }^{\circ} \mathrm{C}$ under argon atmosphere for one hour. The complete alloying process for large amounts of powder can be performed in a short time compared to mechanical alloying. In contrast to other HMS synthesis methods, the proposed process is easy, low cost, quick and efficient in producing alloys of HMS with high phase purity. Therefore, this method can be adopted for commercial production of HMS powder used in thermoelectric technology.

\section{REFERENCES}

[1] B. Poudel, Q. Hao, Y. Ma, Y. Lan, A. Minnich, B. Yu, X. Yan, D. Wang, A. Muto, D. Vashaee, X. Chen, J. Liu, M. S. Dresselhaus, G. Chen, Z. Ren, "High-Thermoelectric Performance of Nanostructure Bismuth Antimony Telluride Bulk Alloy,” Sci., vol. 320. no. 5876, pp. 634 - 638, 2008.

[2] A. J. Minnich, X. Wang, H. Lee,1 M. S. Dresselhaus, Z.F. Ren, G. Chen, and D. Vashaee, "Modeling study of thermoelectric SiGe nanocomposites,” Phy. Rev. B., vol. 80, 15, 2009.

[3] I. Nishida, "Semiconducting Properties of Nonstoichiometric Manganese Silicides," J. Mater. Sci., vol. 7, 435, 1972.

[4] A. W. Searcy, "Predicting the Thermodynamic Stabilities and Oxidation Resistances of Silicide Cermets," J. Am. Ceram. Soc., vol. 40, pp. 431, 1957.

[5] T. Itoh and M. Yamada, "Synthesis of Thermoelectric Manganese Silicide Sintering," J. Elec. Mater., vol. 38, No. 7, 2009.

[6] I. Engstron and B. Lonnberg, "Thermal expansion studies of the group IV-VII transition-metal disilicides,” J. Appl. Phys. vol. 63, pp. 4476, 1988.

[7] B. K. Voroniv, L. D. Dudkin and N. N. Trusova, "Anisotropy of thermoelectric properties in Chromium disilicide and higher manganese silicide single crystal," Kristallografiya, vol. 12, pp. 1527, 1964.

[8] M. Umemoto, Z. G. Liu, R. Omatsuzawa and K. Tsuchiya, "Production and characterization of Mn-Si thermoelectric material," Mater. Sci. For., vols. 343-346. 918-923, 2000 and J. Meta. Nano. Mater, vol. 8. Pp. 918-923 2000.

[9] T. Itoh and M. Yamada, "Synthesis of Thermoelectric Manganese Silicide by Mechanical Alloying and Pulse Discharge Sintering," J. Elec. Mater., vol. 38, pp. 7, 2009 